\title{
Developing a single electronic health care record: better information, care and outcomes in a 'Better care together' world
}

\author{
Colin Brown, FRCP;William Lumb, MRCGP;Tim Reynard, MRCGP; Steve Fairclough
}

Our healthcare economy has met with significant challenges in recent years, with challenges to patient safety, clinical quality, suboptimal patient experiences and major financial challenges. A systematic reorganisation of our clinical services has begun with a clinically led strategy for a very different configuration of clinical services, in a process known as 'Better care together'. New patterns of care and patient flow have been devised, with more emphasis on supporting informed self-care, reducing outpatient follow-up when not really necessary, or providing support through technically supported clinics (telephone, webex). We need to identify patients who are vulnerable before a crisis occurs, to provide early community health and social interventions to limit unnecessary hospital admissions and also to support active early discharge planning and repatriation to homes and the community.

\section{VISION}

Please imagine these different experiences for our patients in the future!

I. Mrs Peacock is 65 years old, lives in a small rural town, has experienced moderate abdominal pains for two weeks and lost one stone in weight. She would like to see her general practitioner (GP), but having telephoned the single point of contact (Care Coordination Centre), which is the central area contact and booking centre, the first available appointment with her GP is seven days away. The contact centre can see GP clinic bookings and is able to make two other offers, including an appointment with a GP Partner in her own practice in five days and a third offer of an appointment with a GP in a neighbouring small town in two days. Mrs Peacock prefers the appointment in two days. The GP at the different practice can provide a consultation for Mrs Peacock, see all her GP Electronic Patient Records (EPR) and Mrs Peacock receives timely, safe and effective assessments and treatment.

2. Colonel Mustard is a 73-year-old who suffered a stroke three months ago, developing a moderate severity right hemiparesis. He had a complicated eight-week inpatient stay requiring extensive rehabilitation before discharge from hospital, but over the last four weeks Colonel Mustard has struggled with worsening mobility with his Zimmer frame and has fallen in the last two days at home. Mrs Mustard calls the single point of contact (Care Coordination Centre) for help and advice. The booking centre telephonist takes details and involves a clinical Care Navigator, who can quickly access Colonel Mustard's EPR and see GP details through Emisweb and also hospital care details through Lorenzo. This includes diagnoses, detail of functional capability and care needs. Instead of a traditional approach of organising ambulance transport to the emergency department (ED) and then another potentially prolonged hospital admission, the Care Navigator is able to mobilise the Integrated Core Team in the community. A physiotherapist and district nurse visit the patient at home within two hours, they have access to all relevant EPR records and care plans and put in place a comprehensive supportive care package over the next two weeks. The outcome is that Colonel and Mrs Mustard receive the support and care they need at home, in a personal setting and a further long inpatient stay with attendant risks, including risks of hospital acquired infection, has been avoided.

3. Professor Plum is a 78-year-old who lives on his own with no family or social support and is admitted with severe leg cellulitis and recent background of poor mobility and poor self-care. Within two days, his cellulitis has improved on intravenous antibiotics, but he is still unsteady on his feet. The Care
Coordination Centre is actively involved in monitoring inpatient journeys in hospital and can plan more rapid assessments and support in a more personal environment. Instead of the current 'risk averse' healthcare processes, where a patient has prolonged inpatient stays involving medical treatments, Professor Plum is discharged promptly to his home where he receives a continued short course of intravenous antibiotics provided by district nurses. $\mathrm{He}$ also receives urgent assessments by physiotherapists and social workers from the Integrated Core Team; Professor Plum receives the extra support he requires. This is a policy known as 'discharge to assess', which reduces hospital stays and reduces time back to functional health within the patient's home environment.

\section{Miss Scarlet is a 26-year-old lady, diagnosed with Crohn's} Disease five years earlier. She had a small bowel resection four years ago, and has been taking azathioprine since then. Her life is simpler and more convenient that previously. Miss Scarlet arranges to have a monitoring blood test with the practice nurse every three months, and then in an evening she has a planned telephone call with her Inflammatory Bowel Disease Specialist Nurse to discuss her health, results and treatment. In earlier days this consultation was provided by telephone, but now she can dial into her nurse clinic using her PAD and have a video consultation with her nurse, using Webex, at a time which is more convenient to her in the early evening. Miss Scarlet no longer has to put up with long waiting times to see the hospital doctor in clinic every few months, which means she loses less time from her selfemployed business. Miss Scarlet has a contact number for her nurse if she needs earlier advice and is comfortable in the knowledge that her nurse will link in with the consultants when needed.

In order to provide this different landscape of 'Better care together' health and social services, we need different groups of healthcare workers, working in different ways and supported by information at point of care. Digital EPR records in GP surgeries, communities and the hospital need to be joined up, so that the relevant information is available at point of care, to support decisions at the next point of care and also joined up with social services. Summary views of other clinical EPRs are currently available in ED, Acute Medical Unit (AMU), Acute Surgical Unit (ASU), and by pharmacists and palliative care teams within Morecambe Bay hospitals. Data and information that need to be shared include messages, documents and care plans. 


\section{HOSPITAL ELECTRONIC CARE RECORDS (EPR): THE LORENZO EXPERIENCE}

University Hospitals of Morecambe Bay (UHMB) provided the 'first of type' NHS deployment of Lorenzo in June 2010 with Patient Administration System (PAS) functionality, documents, notes and forms and test results. As a digital EPR system, Lorenzo offers the ability to record structured digital terms, such as diagnoses, comorbidity, operations, allergies and alerts. Since that time, advanced bed management, Immediate Discharge Summary (IDS) letters, discharge prescribing, ED and Maternity Modules have been added, together with a single ward deployment of inpatient prescribing. Lorenzo is the ubiquitous corporate EPR solution within UHMB and is open on virtually all clinical desktop PCs with a substantial developed library of patient documents and clinical notes and test results in all EPRs.

Over 8,000 clinic appointments per month are now provided without the need for the traditional paper record. This has been enabled by the facility to publish over 40 different types of external test results into Lorenzo EPRs. It is expected that we will see much reduced clinical dependence on the traditional paper record in outpatients by March 2015 .

Further developments in Lorenzo will improve usability through configuration of 'Clinical Charts', which will provide a specialty-specific filtered presentation of documentation and will support 'paperlite' outpatients. Electronic requesting will support test tracking, acknowledgement, and also record who has seen the results. Nursing e-whiteboards have been developed to the specification of UHMB clinicians and have been used on some wards. These link with Lorenzo EPRs, support prompting and noting of clinical assessments, support efficient inpatient care pathways and active discharge planning. If external financial support is provided via successful Tech Fund bids, we can deploy devices around the inpatient estate of UHMB to support clinical care. A third inpatient capability is e-prescribing (IPPMA) which has been deployed initially on a single ward at Royal Lancaster Infirmary (RLI), where the first phase supports oral medications and iv bolus therapy. A second phase of e-prescribing will support iv infusions and medical gases.

In 20 15, Lorenzo users will see significant improvements to the word processor with enhanced performance and 'MS Wordlike' functionality such as 'copy and paste',' 'drag and drop' and toolbars. Voice dictation is supported by Lorenzo in June 2015 (WinVoice Digital Dictation).

Also in 20 15, UHMB will evaluate a mobile offering of Lorenzo used on a tablet device with clinical observations recorded at the bedside, as a prelude to planning how to transform inpatient clinical care onto a fuller digital platform. Data entry will improve with streamlined recording of procedures and alerts and allergies later in 2015, and this will complement the current streamlined entry of problems and diagnoses already in Lorenzo.

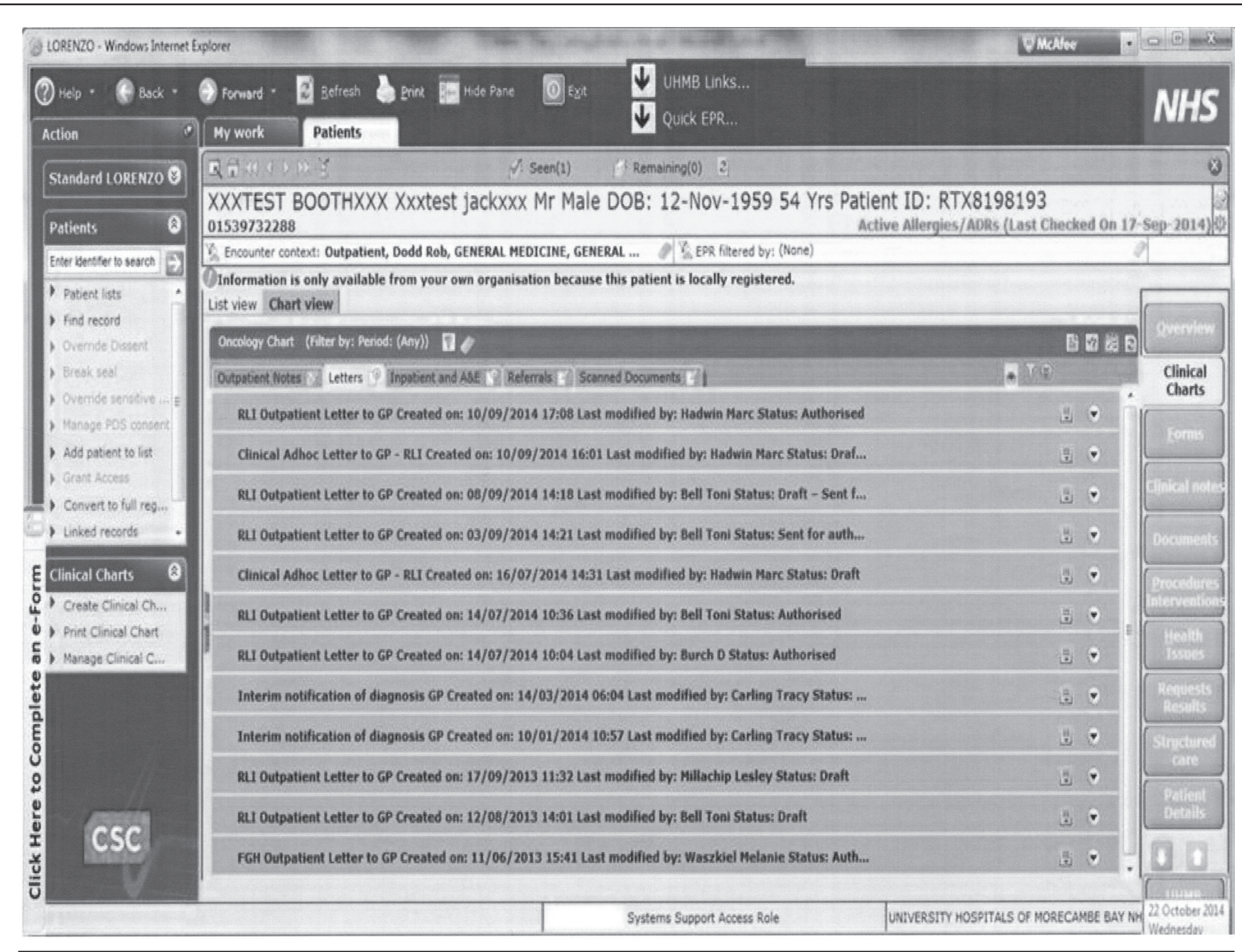

Figure I Clinical Charts in Lorenzo: this gives a 'specialty-specific' collection of relevant forms, notes and letters 
A Theatres module is planned for Lorenzo by September 20 I5. Increased interoperability for Lorenzo will facilitate integrated views of care records across primary and secondary and community care. Increased integration of the Medical Interoperability Gateway (MIG) in June 2015 will provide Lorenzo Viewing (Lorenzo Summary for GPs in Emisweb), community record viewing and also integrate with Docman Hubs.

Paperlite inpatient clinical care on the wards is expected to complete phase one by April 2017, including full rollouts of e-test requesting, e-prescribing, e-nursing whiteboards and nursings care plans. Phase two will complete this process with full medical documentation, integrated care pathways, task management and referrals.

\section{GP ELECTRONIC PATIENT RECORDS: THE EMISWEB EXPERIENCE}

General practices now all use the same patient record system, EmisWeb, which provides GP clinicians with opportunities for collecting patient information in increasingly standardised formats. Local clinical initiatives are easier to support with agreed clinical data sets, which are collected in standard templates. The whole health community benefits from a single secure network linking all healthcare buildings across Cumbria and North Lancashire. This secure infrastructure layer provides the platform for secure interoperability of the health community's patient record systems.

There is a significant infrastructure development in progress across Cumbria Primary Care with the deployment of a full virtualised desktop being delivered to every desktop device in every GP practice. This will ensure every GP has a desktop that follows them across the whole Cumbrian geography, which in practice means that the GP's individual PC configuration is available everywhere, but delivered securely to them based on their logon credentials. This gives the GP a standard user experience wherever they are whilst at the same time reducing logon times and cutting maintenance overheads.

\section{INTEROPERABILITY, MIG, STRATA, DOCMAN, ADVICE AND GUIDANCE}

The electronic sharing of patient information between clinicians is now commonplace in many areas locally. This happens through a small number of interfaces which allow a clinician to record consent and then see a view of certain information from the GP system via their own clinical system. What they can see depends on who they are, where they are and the role they have in the delivery of the care of that patient. An increasingly diverse and growing stream of documents is also flowing out of UHMB into general practice via either the MIG interface or the Docman Electronic Document Transfer Hub.

\section{IDS (discharge summary) letters}

Immediate discharge summaries are now sent automatically to GPs, promoting timely delivery (currently, around 85\% are delivered with 24 hours of discharge). The structured template provides the clinician with the opportunity of adding additional appropriate information. The same template will, in time, allow the completeness of the IDS to be audited.

\section{Outpatient letters (Docman and MIG)}

Some other documents, such as endoscopy reports and A\&E letters, are already being sent out through the MIG as with IDS letters. Outpatient letters are currently being sent from gynaecology to Lancaster GPs with a system called Docman, and the number of specialties involved in this will increase in coming months.

\section{Summary views (GP Summary, Medicines, End of Life or supportive care)}

The most obvious evidence of shared records is currently to be seen in ED and the Medical Assessment Unit. Here, clinicians can, with consent from the patient, view a medical history summary, medication and allergies. Pharmacists have found the latter two elements extremely valuable in their medicines 'reconciliation' work, ie making sure that patients admitted to hospital are maintained on the drugs they were taking in the community. The number of requests for medication information to GP practices has fallen by up to $99 \%$.

Other new developments have allowed us to share the clinical terms in the GP record as well as the associated free text. The viewing can now read the additional notes critical to a more complete understanding of the patient and their health needs.

We have implemented the Electronic Palliative Care Coordination System (EPaCCs). The clinician can see that a palliative patient has a DNA CPR decision and can also see that the GP has made a note, for example, that the daughter was supportive of this decision. The EPaCCs implementation could be the first of many more specialised data sets which are now simpler to deliver. These could support cross boundary working for patients with chronic health needs. The EPaCCs project will continue to extend this sharing into the community nursing records and the implementation of a new electronic patient record system in both hospices will further enhance the available information for this vulnerable group of patients.

\section{Advice and guidance}

A locally developed 'Advice and Guidance' system has been clinically defined. Clinical conversations can now take place, which support primary care physicians and support referrals management: these conversations can be retained as part of the clinical record in Lorenzo and Emisweb. Overall, this has led to a significant reduction in traditional referrals to secondary care and improves the preparation of patients referred through earlier assessments, tests and treatments.

\section{Discharge planning via Strata}

UHMB faces a significant challenge in actioning appropriate and timely patient flow via referrals within our complex health community, including social and community care and mental health services. The historic challenges are largely due to ineffective paper-based systems and a lack of directories of services to support patient needs.

Cumbria Clinical Commissioning Group has provided a digital solution ensuring integration and transparency to meet the triple aims of improved data quality, timeliness of patient flow and effective use of resources and cost reduction. Strata Pathways manager system allows hospital staff to send an electronic patient assessment to adult social care. The system will match the patient needs against available resources. The Strata system currently works in inpatient areas and Occupational Therapy and significant benefits are being seen already. 


\section{Test results}

Over 50 different types of test results have traditionally produced paper reports, which are filed in the hospital paper record. If these systems are plugged into the hospital network, with results files which have sufficient patient identifiers, it has been possible to publish the result into the individual patient's EPR. Over 40 different types of test results have been captured into the Lorenzo EPR so far.

\section{‘BETTER CARE TOGETHER’ CLINICAL STRATEGY}

\section{Ambition}

The vision is to transform the care provided in Morecambe Bay, to create a seamless experience for patients, whether they are receiving care in the home, community or hospital. Our new model of care needs to be centred around the patient rather than the care setting and to focus on the quality of the services being delivered, rather than the organisation that delivers them.

We have entered into a vicious circle of spending too much money on hospital care and leaving insufficient resources to invest in primary and community services. This has led to more people being admitted unnecessarily to hospital, resulting in increased spend in the most expensive part of our system and often poorer experience for our patients; our care is costing more to provide than we have available. We recognise that the populations we serve, like those across the country, are likely to see big increases in demand for health care due to factors such as lifestyle, age and the increasing prevalence of longterm conditions. Similarly, we understand there are demographic and geographic factors that make Morecambe Bay unique.

\section{The ambition is to create a sustainable health and social care system that provides the best care possible based on five objectives}

I Integrated models of care across the local health economy that meet agreed local and national clinical standards and can deal with changes in our population and their needs.

2. A system which recognises the specific geographic and demographic characteristics of our area and enables the population to access the most appropriate settings of care for their health needs within reasonable travel times. The emphasis will be on providing these services in, or as close as possible to, people's homes.

3. A system which encourages the improvement of health and wellbeing, clinical outcomes and patient experience, in a way which is sustainable.

4. A flexible, integrated and productive workforce across our health economy. This approach will enable staff to develop continuously, realise their potential and achieve greater job satisfaction.

5. A future healthcare system for our area that makes best use of the money and resources available.

\section{Transforming clinical models of care}

A future clinical care model has been re-designed so patients receive care outside of a hospital setting, where safe to do so. We need to consider what these changes mean for the delivery of services in our three hospitals in Morecambe Bay. To support the development of our Out of Hospital model of care, our clinicians have developed design principles and their preferred clinical models for Episodic Care, Complex Care, Unscheduled Care, Children and Young People's Care and Maternity Care. The principles are set out below:

I. Our services are of good quality, delivering safety, equity and continuous improvement in outcomes.

2. Our system is sustainable both financially and clinically and is flexible to meet future demands.

3. Our design will build on what is already working well and recognises that some of what we are currently doing has to change.

4. We will encourage our patients to take more responsibility for themselves and we will take account of their views in our service design.

5. All design options will be realistic and based on evidencebased best practice.

6. Care will be provided in the right place and at the right time. There will be more care delivered out of hospital and some small volume specialty work may not be viable to provide locally.

7. Services will be provided in an integrated way with shared records, fewer handoffs and easy navigation.

8. We will manage patients proactively and patients will know who is responsible for their care.

9. Morecambe Bay will be an attractive place to work and our workforce will be developed and encouraged to deliver services which are in the best interests of patients.

\section{Delivering care in a radically different way}

The ambition is to reduce the reliance on our hospitals and increase the scale and quality of care provided in the community. Our Out of Hospital model will enable us to make this transformational change (see figure 2).

\section{Five elements of the new Out of Hospital strategy}

The Integrated Core Team is a multidisciplinary team of care professionals and third-sector staff, addressing all of the care needs for a population of patients. The essential function is to proactively manage patients, reduce hospital admission and allow early hospital discharge and recovery at home.

The Care Coordination Centre provides a single health and social care hub for both professionals and patients. This team will work with the core team and care navigators to place patients in the right service for their needs.

Integrated Rapid Response Team is deployed to supplement the core team, with the aim of reducing unnecessary hospital admissions. The team makes a rapid assessment of the patients' medical, nursing and care needs, delivering a package of care before handing the patient back to the core team for recovery.

Community-based Specialist Services, where a set of care pathways are defined and specialist advice can be secured in an out of hospital setting. The elements of this community service are direct patient care, professional education and responsibility for population outcomes.

The Referral Support System will encompasses the accessing of specialist advice and guidance, improve access to diagnostic investigators, develop care pathways across specialties, including decision aids, making these pathways clearly available to those involved in patient care. 
These elements fit together in a coordinated system of care with the needs of 'whole person' (physical, psychological and social) at the centre. This future model of care has at its heart a commitment to support a more integrated health and social care service in the coming years.

\section{‘BETTER CARE TOGETHER’ IM AND T STRATEGY}

The IM and T strategy in 'Better care together' support

Bettercare future configuration of clinical care in Morecambe Bay. The most complex clinical services model is the Out of Hospital model, and so this was used primarily to underpin an 'IT systems design' which will support integrated care out of the hospital setting. The challenge for us in Morecambe Bay is to get three established IT systems (Lorenzo, Emisweb and, in the social services, LiquidLogic) integrated with one another, with Rio as a future new EPR system supporting mental health services in Cumbria.

Significant progress has been made with connections between Lorenzo and Emisweb, including views of GP records through Lorenzo EPRs, electronic delivery of letters from Lorenzo into Emisweb and sharing of clinical messages. Planning was undertaken to assess what is needed to underpin integrated clinical care, to develop an IM and T Future State Model, which supports the clinical service pathways. Four groups of overlying solutions were identified.

\section{Initial Access systems}

Each locality will need a single central access point for both patients and also healthcare workers in the Integrated Core Team. Access to clinical services will be via a number of modalities including telephone, online booking and in person. This will provide better contact centre options and triaging, as illustrated by the case of 'Mrs Peacock' at the start of the paper. Initial access systems will also include knowledge bases for patients to provide support for self-care and self-management, illustrated by the needs of 'Miss Scarlet', but also Directories of Services and knowledge bases and decision aids for healthcare staff, to support 'care navigators' in defining the optimal care pathway for patients according to their needs. Referrals support will also be offered here via 'Advice and Guidance', and referrals management supported through increased use of Strata system. In an early phase, we can pilot some clinical care pathways in some localities, to gain confidence in developing care plans, presenting them seamlessly to successive healthcare professions across different organisations, so that the patient receives logical, ordered and connected healthcare, with professionals having the right clinical information at point of care to provide optimal and timely decisions and clinical care.

\section{Core systems}

A single EPR allows the clinician or social care worker to view what is needed to provide appropriate decision making. Expansion of the MIG device, Lorenzo and Emisweb will support extensive sharing of letters, reciprocal views of Lorenzo

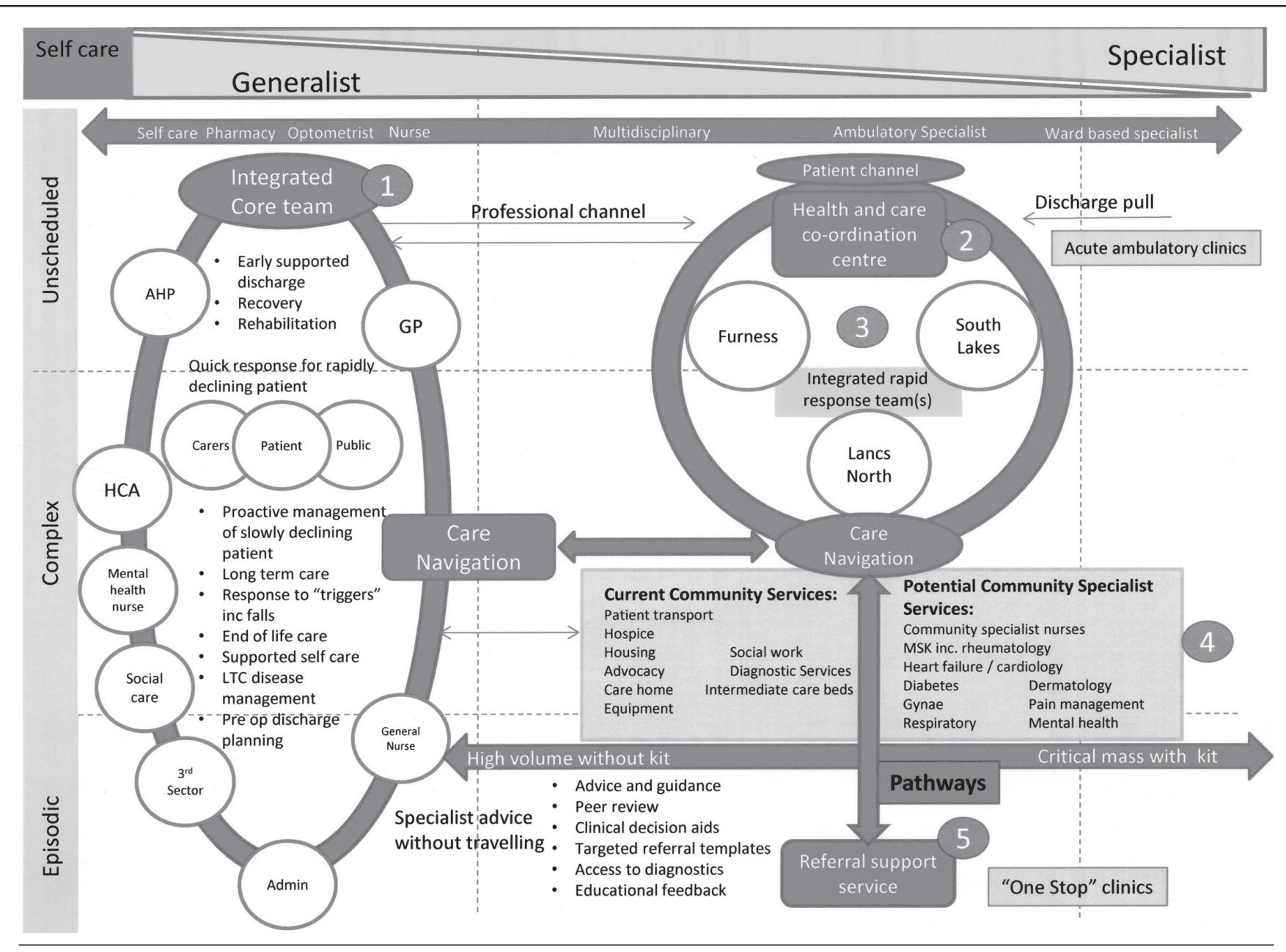

Figure 2 Out of Hospital clinical services model 


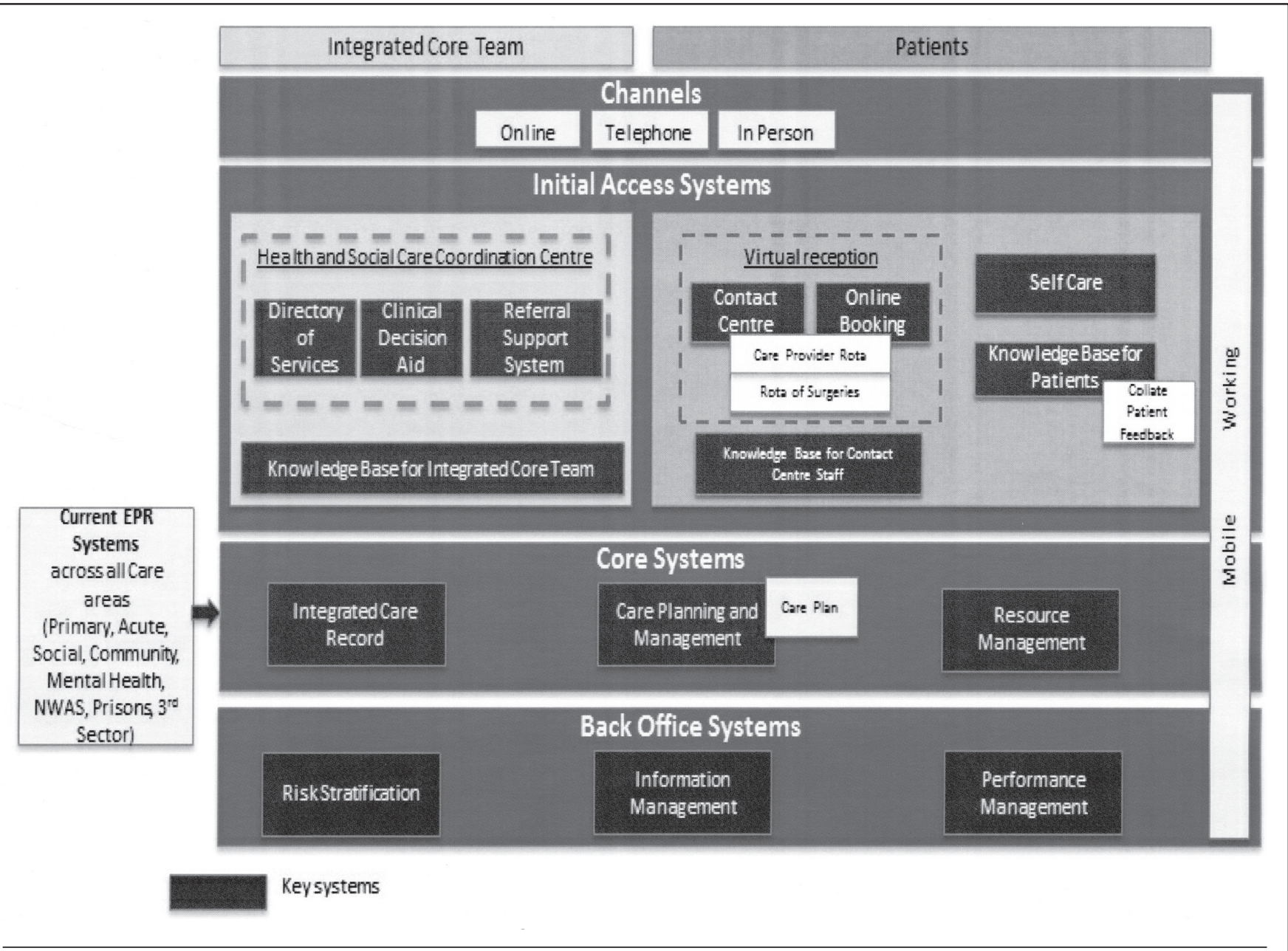

Figure 3 Future state IM and T systems and capability

within Emisweb and increased use of bi-directional clinical and business messaging between EPRs. Sharing of structured data, messages and care plans will replace many letters. There is currently a Lancashire initiative to develop a 'sharing platform' known currently as LPRES. This device provides two sharing capabilities. Firstly, a healthcare sharing platform will allow organisations to publish data and information to the platform, consistent with predefined information governance sharing agreements. Other organisations will be able to access and share this information. In this way, we can support connected care pathways that involve UHMB, Blackpool, Preston and East Lancashire hospitals. Secondly, there will be support for personal citizen records to be held and developed. In this way, citizens and patients can build up their own record content, which would include information provided to them from healthcare professionals, their own healthcare data, such as weights, peak flows and blood sugar measurements, and also data from gyms and other parts of their lives. Citizens and patients can then choose who they share this information with, which may include other healthcare professionals and charities and the third sector.

A Care Planning and Management system will also be needed to sit on top of clinical EPRs, to provide cross-sector and crossorganisational pathways of care by developing care plans for health and social care staff. Care Pathways will be defined, agreed and adopted by local clinicians and the Care Management system will support the clinical pathway according to 'systems rules' operating in the background. This system will also function to allocate and manage the resources required for these care pathways, including care professionals.

\section{Back Office systems}

We will need Business Intelligence and reporting capability to provide a number of functions. In the future we need to recognise contacts with healthcare and voluntary organisations and be able to identify the frail and vulnerable at an earlier pre-crisis point. We will then be able to provide lower tech interventions at community level to support patients in their own homes and communities and to avoid unnecessary costly hospital admissions. We also need to provide operational reporting and performance management information and to manage effectiveness of care and clinical and business outcomes. We will build on currently available systems such as Microsoft Reporting services (Analyzer) and Qlickview.

\section{Infrastructure solutions}

We already have mature IT networks in Cumbria and North Lancashire. There is a modern data warehouse at Furness General Hospital (FGH), which currently provides support for the 'single source of truth'. A new data warehouse is also being set up at Penrith and there are plans to provide modern data warehouse capability at Lancaster. It is important to provide a single-user desktop experience, whereby it doesn't matter where the clinician is geographically located so IT systems present to the user and work in the same way. A more mobile 
clinical workforce may develop and the Cumbria initiative to provide virtual desktops across all the GP community greatly supports this. It is also important that Cumbria organisations converge towards the same single active directory, where users have the same email domain and this then supports mail, communications and other technologies, such as visual Webex and audio Jabber. In so doing, remote but secure consultations are supported. This planning is important in generating solutions for improved, consolidated and connected clinical services in isolated communities.

\section{CLINICAL INFORMATION CULTURE}

EPRs have been established in general practice since the early 1990s. For many years, hospital clinicians have benefitted from the refined and accurate GP records, with extracts available in condensed form in referral letters and containing key data such as significant previous diagnoses and problems, allergies and medication lists. This data has been 'cleaned up' over many years and whilst UHMB Lorenzo EPRs are mostly 'light' on structured clinical data currently, there is a need to build data content of EPRs, so that structured clinical terms which are episode-specific link with one particular episode of care (eg episode of pneumonia) whereas a valuable co-morbidity continuing diagnosis (such as COPD) is noted to relate to all encounters. Lorenzo is a proper digital EPR system, supporting record organisation into a 'problem-orientated' record. To exploit this, there is a need for clinicians to input key digital 'structured clinical' terms, such as problems, symptoms, diagnoses, comorbidity, alerts and

allergies, operations and procedures. These data terms should be inputted during usual processes of healthcare at "point of care' so that becomes normal clinical behaviour and is not a separated function.

In 2013, the Trust board approved a plan to change outpatient services so that more than $80 \%$ of clinical appointments and consultations would be provided without traditional paper notes. Virtually all clinical specialties have now defined and been provided with their clinical content requirements. Full clinical adoption of 'paperlite' clinics is expected by March 2015.

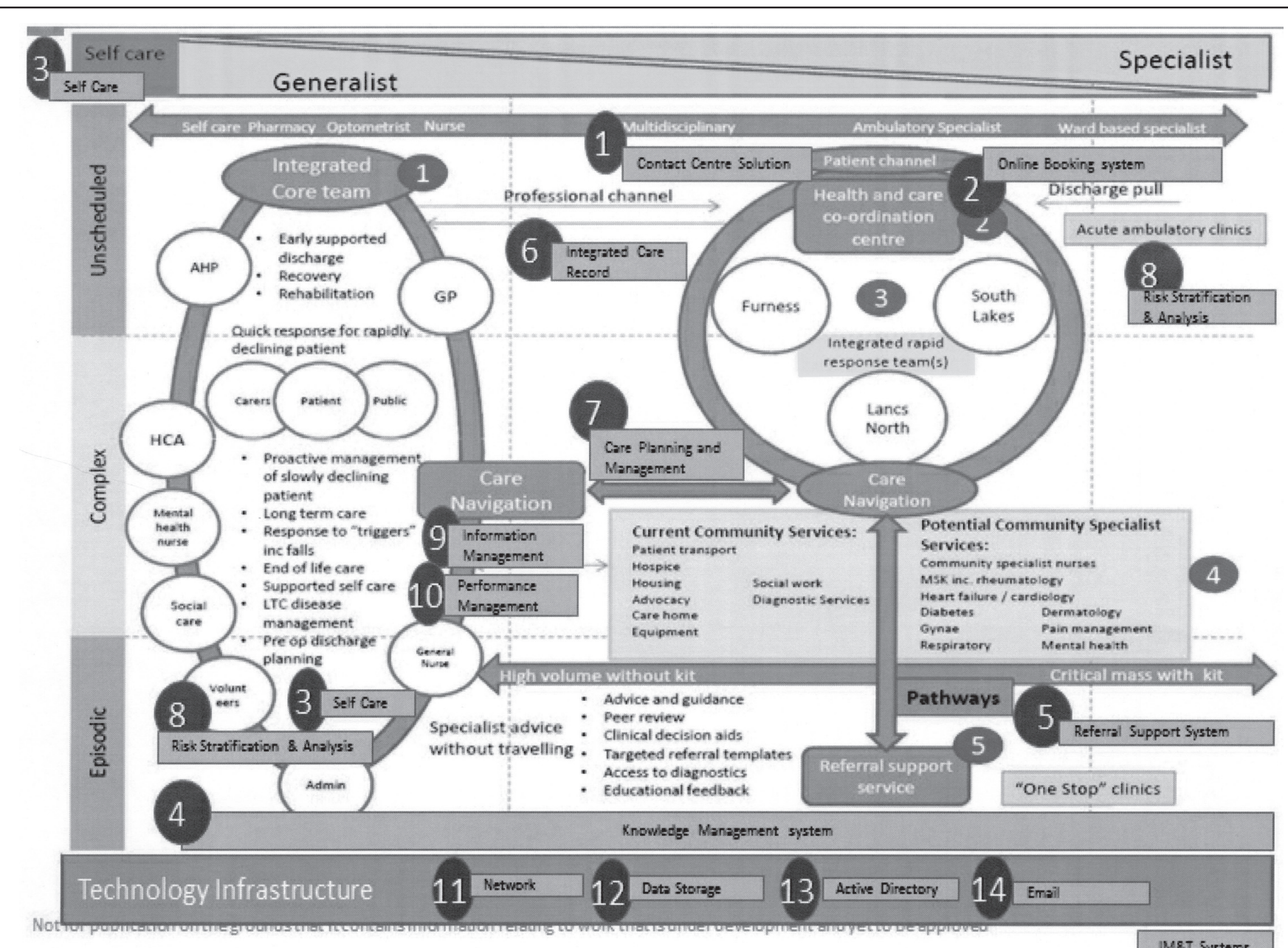

IM\&T Systems 
As well as the obvious challenge of obliging colleagues to move to a paperlite platform there is the challenge to change the mindset of colleagues from simply CONSUMERS of information in EPRs, to being active participants in EPRs and generating new information in EPRs, which inform the current and future episodes of healthcare. Clinicians need to become active PRODUCERS of information.

The people best placed to record accurate details of why a patient is seeking healthcare are the clinicians. We are more likely to obtain accurate information about diagnoses, comorbidity and 'acuity of illness', case-mix in outpatients, clinical risks, allergies and alerts if this information is considered, defined and captured by clinicians. This would best be done at point of care on inpatient ward rounds, or after an operation or outpatient consultation, led by consultants. In so doing, we will be recording the clinical reason for that episode of care, and generating a 'problem-orientated EPR record'.

When structured clinical terms are generated by the clinician, these terms are then available for secondary uses and can be re-used for that or future episodes of care in ways that free text or traditional letters cannot. The primary purpose of generating new structured data in EPRs is to inform current healthcare and is best done at point of care. However, this information is available for many secondary uses:

- Clinicians re-using data. These terms inform future healthcare and can be used, re-used and re-used by successive clinical users to populate forms, clinical notes and letters with diagnoses, co-morbidity and alerts, in current and future episodes of care.

- Clinical coding. Clinical coders are trained to look at the clinical record and to utilise data on diagnoses, problems and co-morbidity. UHMB mortality has substantially decreased since 201 I, partly due to improved clinical coding.

- Activity and contract delivery. This is supported by clinical coding and hospital episode data, such as admissions, discharges, outpatient appointments.

- Quality Assurance (QA) and Clinical Audit. Audit of paper-based records is problematic, with incomplete retrieval of paper records. Electronic data, if described in structured terms, are very easily retrievable. When clinicians routinely and systematically record structured clinical terms in EPR records, then QA and auditing at individual, specialty, divisional and corporate levels becomes very straightforward.

- CPD and individual performance. Already some surgeons have seen the benefit of using structured clinical terms, whereafter database searches provide information on personal workload and experience and outcomes for use in CDP logs and to support appraisal and re-validation.

- $\mathrm{R}$ and $\mathrm{D}$. Any patient in a research trial can be flagged with an alert in the EPR record, so that those patients needing episodic clinical care have their research trials noted to clinicians. EPR can also be used to identify patient cohorts suitable for research and to facilitate monitoring of future outcomes.

- Flow of information across organisations. The ultimate aim, of course, is a single patient EPR accessible to all healthcare workers where needed.
Data is captured, stored and reported through the UHMB Single Source of the Truth (SSotT) data warehouse to ensure data quality in reporting. The delivery of true clinician-centric reporting has historically been difficult to achieve within acute hospitals (persuading acute hospital clinicians to take responsibility for recording their own electronic data has historically been very difficult) and has required excessive use of manual audit systems. Advances have been made over recent years and the Trust subscribes to the HED benchmarking system that enables self-service tailored reporting to support medical revalidation. These systems rely on data from financebased reporting regimes and the allocation of workload against individual clinicians is rules-based, which can be frustrating for the clinician wanting to understand exactly what work they undertook with what outcome. The development of a clinically rich and clinically authored EPRs will enable true patient- and clinician-centric reporting with reporting focused on outcomes. Clinicians have historically been suspicious that the data from these systems, which is used for finance-based systems, is being inappropriately used to link quality with productivity.

The development of a clinically rich dataset potentially offers us a great opportunity to build upon this model through the development of an Analytical Unit that can support clinical outcomes analysis and research.

Analysis of pathways of clinical care for patients is possible at a much deeper level than previously possible with a predominantly transaction-based record. All UHMB Clinical Divisions now have a Divisional Business Intelligence Analyst to support clinical and operational delivery and initial feedback has been extremely positive.

\section{Implementing the electronic patient medical record}

We also need to recognise any barriers that discourage active engagement of clinicians with EPRs and look for opportunities, to promote a 'clinical information culture'. So, what can be done? The fundamental requirement is cooperation between clinicians working with EPR contents experts.

- improving the understanding for clinicians regarding the importance of data input into EPRs

- making it easier for the clinician to enter data

- exploiting existing capability in Lorenzo such as Clinical Charts to give a specialty filtered view on documents and request tests

- configure the Snowmed browser to be easier to use develop speciality favourites folder for Snowmed terms

- develop EPR clinical content with users to support care processes via forms, notes, letters and care plans

- develop different practices which incorporate management of clinical information at the heart of our clinical work. This may include standard operating procedures for ward rounds and outpatient clinic consultations

- use information analysts working with clinicians to identify specialty information needs and help to plan for how these are met

- develop information reporting Business Intelligence systems which support measuring clinical outcomes and clinical management of services. 


\section{Implementing the electronic patient nursing record}

There are some good examples of nurses using electronic systems to support modern healthcare. The Infection Prevention Nurse (IPN) team input infection alerts into Lorenzo EPRs and this drives a working group and workflow. Midwives use the Maternity module of Lorenzo, which has been designed to support governanced, safe clinical care. Nursing staff on ward 37 at RLI use the IPPMA module of Lorenzo, which supports safer drug prescribing and dispensing and ward 6 staff at FGH use an electronic whiteboard to support earlier clinical assessments and supported planning for earlier discharges. There are also processes which have been developed to support CQUIN care and also Acute Admission Assessments and Assessment of the Frail Elderly. Currently, external financial support has been sought so that a rollout of e-whiteboards, IPPMA and also test requesting around all wards and departments in UHMB is enabled. Nursing care has been heavily dependent on complex paper records and UHMB has just been supported with a Digital Fund bid of $\mathrm{EIm}$ to modernise with use of care plans in Lorenzo and for recording of observations in the near future. The key to success is the development of appropriate clinical content which is configured and supports nurses, nursing care and workflow, in the same way as it supports the work of medical staff

\section{SUMMARY}

The NHS has a vision and strategy to be 'paperless' by 2018. Morecambe Bay has made very significant progress in adopting electronic patient records in hospitals and in general practice and the community. Sequential adoption of digital support for clinical care will support the move away from paper.

It is the purpose of the informatics community, to provide the digital support required for clinicians and social workers, in connecting health and social care across sectors and across organisations. Increasingly, we will need to share care plans, messages as well as letters, between EPR systems in different settings. Hopefully, this paper informs clinical colleagues as to how this will be achieved.

Out of the ashes, the 'phoenix' of a healthy Morecambe Bay will arise. This is everyone's business and it will need all colleagues in local health and social services to actively take part in bringing this approach into our everyday Morecambe Bay healthcare life.

Colin Brown is Chief Clinical Information Officer, University Hospitals of Morecambe Bay (UHMB); William Lumb is Chief Clinical Information Officer, NHS Cumbria;Tim Reynard is Chief Clinical Information Officer, NHS North Lancashire; and Steve Fairclough is Chief Information Officer, UHMB. 